\title{
Electro-acupuncture for irritable bowel syndrome patients: study protocol for a single-blinded randomized sham-controlled clinical trial
}

Linda L. D. Zhong ${ }^{1,2^{*}}$ (D), Tsz Fung Lam ${ }^{1,2}$, Wei Yang ${ }^{1,2}$, Ya Zheng ${ }^{1,2}$, Zipan Lyu $u^{1,2}$ and Zhaoxiang Bian ${ }^{1,2}$

\begin{abstract}
Background: Irritable bowel syndrome (IBS) is one of the most common functional gastrointestinal disorders in clinical practice. IBS diagnosis is based on symptoms defined by abdominal pain or discomfort associated with defecation or changes in bowel habits. Gut-brain interaction caused by stress or depressive emotion is one of the essential pathologies. Acupuncture has been used for the treatment of internal medicine, including digestive disorders and depressive disorders in Chinese medicine. This study aims to determine whether electro-acupuncture could have significant benefits than sham acupuncture for IBS.

Methods/design: This is a single-blinded randomized sham-controlled clinical trial with two arms. A total of 120 IBS patients will be recruited. After a 2-week run-in period, eligible subjects will be randomly assigned to one of two arms, acupuncture (AC) arm and sham acupuncture (SAC) arm. Each eligible subject will go through a 2-week run-in-period, 6-week treatment period, and 6-week follow-up period. Five visits in total were scheduled for each subject in week 0, week 2, week 5, week 8, and week 14. The outcomes would be measured with (1) IBS-SSS, (2) Hamilton Depression Rating Scale (HAMD-17), (3) Clinical Global Impression-Severity (CGI-S), (4) Self-Rating Depression Scale (SDS), and (5) IBS Quality of Life (IBS-QoL).
\end{abstract}

Discussion: The study will compare electro-acupuncture with sham acupuncture to explore the feasibility of electro-acupuncture in improving IBS symptoms.

Trial registration: ClinicalTrials.gov NCT04387383. Registered on 13 May 2020

Keywords: Irritable bowel syndrome, IBS, Electro-acupuncture

\footnotetext{
* Correspondence: Idzhong@hkbu.edu.hk

'Hong Kong Chinese Medicine Clinical Study Centre, Hong Kong Baptist University, 3F, Jockey Club Chinese Medicine Building, 7 Baptist University Road, Kowloon, Hong Kong, SAR, P. R. China

${ }^{2}$ School of Chinese Medicine, Hong Kong Baptist University, Jockey Club Chinese Medicine Building, 7 Baptist University Road, Kowloon, Hong Kong, SAR, P. R. China
}

(c) The Author(s). 2021 Open Access This article is licensed under a Creative Commons Attribution 4.0 International License, which permits use, sharing, adaptation, distribution and reproduction in any medium or format, as long as you give appropriate credit to the original author(s) and the source, provide a link to the Creative Commons licence, and indicate if changes were made. The images or other third party material in this article are included in the article's Creative Commons licence, unless indicated otherwise in a credit line to the material. If material is not included in the article's Creative Commons licence and your intended use is not permitted by statutory regulation or exceeds the permitted use, you will need to obtain permission directly from the copyright holder. To view a copy of this licence, visit http://creativecommons.org/licenses/by/4.0/ The Creative Commons Public Domain Dedication waiver (http://creativecommons.org/publicdomain/zero/1.0/) applies to the data made available in this article, unless otherwise stated in a credit line to the data. 


\section{Background}

Irritable bowel syndrome (IBS) is one of the most common functional gastrointestinal disorders in clinical practice. The diagnosis of IBS is based on symptoms defined by abdominal pain or discomfort associated with defecation or changes in bowel habits [1]. Recent pooled population-based meta-analysis indicated that the global prevalence of IBS was approximately $11.2 \%$ [2], and an early epidemiological study in Hong Kong showed that $13 \%$ of males and $21 \%$ of females in Hong Kong suffered from IBS [3]. To date, the treatment for IBS is unsatisfactory. Moreover, people with IBS frequently suffer from anxiety and depression, which can worsen the symptoms. Of those who do seek treatment, research has found that 54 to $94 \%$ have a psychiatric disorder such as an anxiety disorder or depression [4-6]. Up to the present, there has not been a satisfactory treatment of IBS in pharmacological approach nor complementary medicine [7]. In China, antibiotics, antispasmodics, and secretagogues are used to treat IBS, but the effectiveness varies [8]. Non-medication treatments such as dietary/ nutritional and psychobehavioral therapy can be the alternatives; however, the requirement of high adherence may decrease the rates of treatment continuation $[9,10]$. Acupuncture can be a more convenient option for patients with IBS as the treatment does not require daily intake of medication nor frequent clinical visits.

Acupuncture has been practiced empirically in China for several millennia, and it is increasingly accepted by practitioners and patients worldwide, especially during the last three decades [11, 12]. Currently, acupuncture treatment for IBS is a research hotspot in alternative medicine. A trial of acupuncture in 233 patients with IBS published in 2012 showed acupuncture improved their quality-adjusted life years [13]. Other reviews indicated that acupuncture was effective for IBS global symptoms [14, 15]. Research showed that electroacupuncture had the same effectiveness as medication in decreasing diarrhea on IBS patients with additional improvement on the consistency of stool and visceral pain symptoms [16]. Besides, it is well demonstrated that acupuncture has broad therapeutic benefits in treating various psychiatric disorders. A recent meta-analysis indicated that acupuncture treatment was effective in alleviating various depressive symptoms [17].

A cohort study concluded that IBS patients had an increased risk of developing depressive disorder [18]. At the same time, the disorder is also a risk factor of IBS; therefore, the intervention on depression is critically important. Among the previous researches, abdominal points are most frequently selected and used as core points for IBS treatment. However, these points mainly relieve IBS local symptoms such as bloating and visceral pain [19-22]. Scalp points can be used in combination with the abdominal ones to treat global symptoms like headache, fatigue, or mood change. We may infer that scalp-abdominal point acupuncture is an effective treatment option for overall symptoms of IBS. However, there is no available clinical research applying multiple scalp points in the treatment nor demonstrating this conclusion [23]. Thus, we designed this trial with scalpabdominal acupoints to be the fixed and core points and hypothesized that acupuncture could produce more remarkable clinical improvement compared to sham acupuncture for IBS symptoms.

\section{Objectives}

To test the hypothesis, a single-blinded, randomized, sham-controlled trial is designed to determine whether acupuncture could have significant benefits than sham acupuncture for IBS.

\section{Materials and methods Study design}

This is a single-blinded randomized sham-controlled clinical trial with two arms. A total of 120 IBS patients will be recruited. The study will cooperate with Hong Kong Baptist University and University of Toronto. After a 2-week run-in period, eligible subjects will be randomly assigned to one of two arms, acupuncture (AC) arm and sham acupuncture (SAC) arm. Each eligible subject will go through a 2-week run-in-period, 6-week treatment period, and followed by a 6-week follow-up period. Five visits in total were scheduled for each subject in week 0 , week 2 , week 5 , week 8 , and week 14 . The participant flowchart is listed in Fig. 1, and the participant timeline is listed in Fig. 2.

\section{Participants}

\section{Diagnostic criteria for IBS (Rome IV) [24]}

Recurrent abdominal pain, on average, at least 1 day per week in the last 3 months with symptom onset at least 6 months, associated with two or more of the following criteria: (1) related to defecation, (2) associated with a change in frequency of stool, and (3) associated with a change in form (appearance) of stool.

\section{Classification of subtypes}

After diagnosis, participants will be given a questionnaire with the Bristol Stool Form Scale (BSFS) to classify their subtypes. The study includes all IBS subtypes (Additional file 7).

\section{Inclusion criteria}

Patients will be included if they have all the following at baseline and during the 2 -week run-in period:

1) Fulfillment of the Rome IV criteria for IBS 


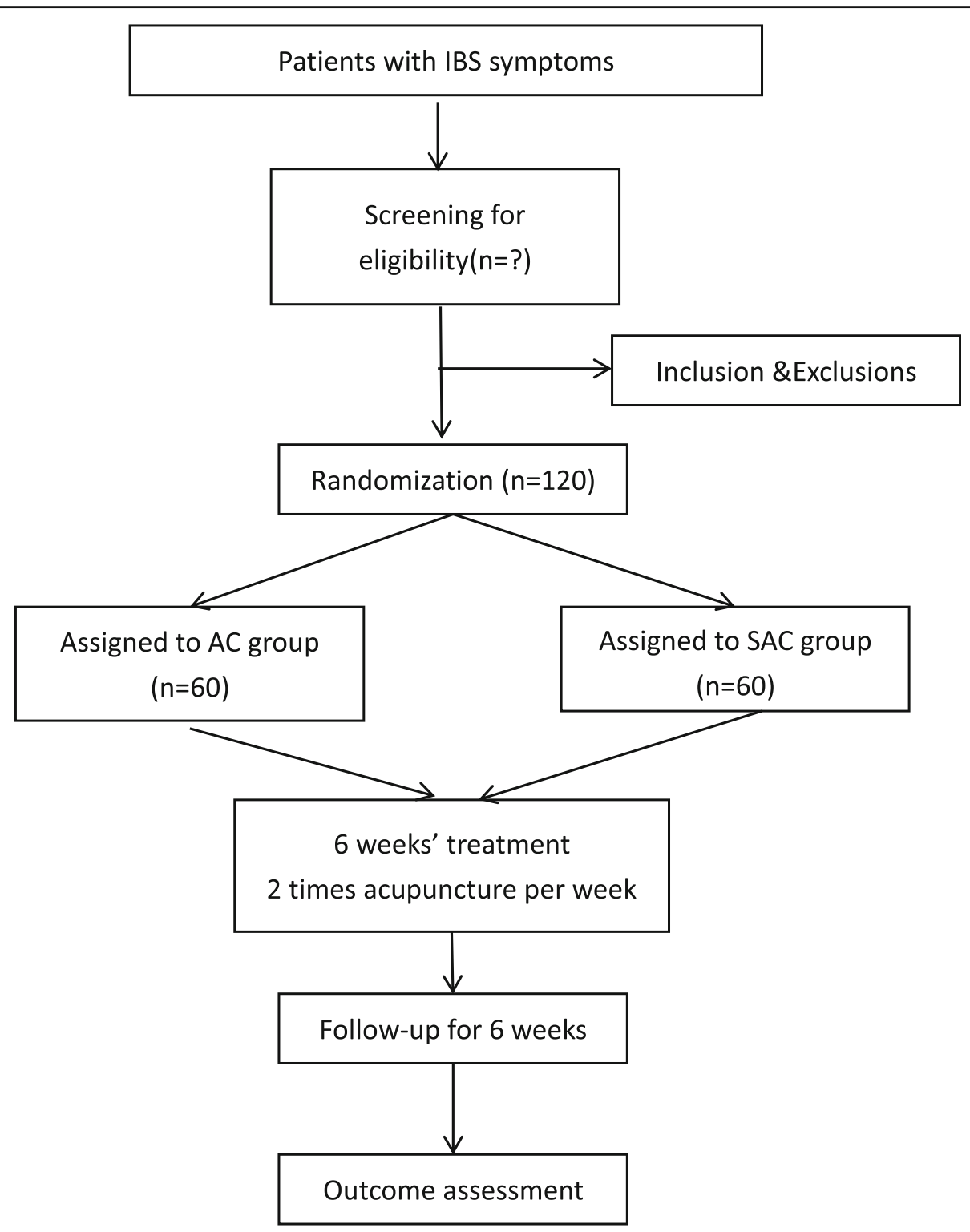

Fig. 1 Participants flow chart

2) Age of 18 to 65 years (inclusive)

3) IBS Symptom Severity Scale (IBS-SSS) (Appendix 1) $>75$ points (a range of $0-500$ points of VAS on five questions)

\section{Exclusion criteria}

Patients will be excluded if they have one or more of the following:

1) Pregnancy or breast-feeding

2) Medical history of inflammatory bowel diseases, carbohydrate malabsorption, hormonal disorder, known allergies to food additives, and/or any other serious diseases

3) Unstable medical conditions
4) Unstable mental condition or with a history of mental illness

5) Patients who have received acupuncture treatment in the last 3 months or took concomitant medication with effects of gastrointestinal motility or visceral sensation, such as antidiarrheal agent, antidepressant, narcotic analgesic, and anticholinergic

6) Alcoholism or drug abuse in the past 1 year

7) Having needle phobia

\section{Withdraw from the trial}

Participants will be allowed or asked to drop out from the trial if they: 


\begin{tabular}{|l|l|l|l|l|l|}
\hline \multicolumn{5}{|l|}{ STUDY PERIOD } \\
\hline & Enrolment & Allocation & \multicolumn{2}{l|}{ Post-allocation } & Close-out \\
\hline TIMEPOINT* & 0 & 2 & 5 & 8 & 14 \\
\hline ENROLMENT: & & & & & \\
\hline Eligibility screen & $\times$ & & & & \\
\hline Informed consent & $\times$ & & & & \\
\hline Allocation & & $\times$ & & & \\
\hline INTERVENTIONS: & & & & & \\
\hline AC arm & $\times$ & $\times$ & $\times$ & $\times$ & \\
\hline SAC arm & $\times$ & $\times$ & $\times$ & $\times$ & \\
\hline ASSESSMENTS: & CGI-S & & & & \\
\hline$H A M D-17$ & & $\times$ & $\times$ & $\times$ & $\times$ \\
\hline SDS & & $\times$ & $\times$ & $\times$ & $\times$ \\
\hline IBS-SSS & $\times$ & $\times$ & $\times$ & $\times$ & $\times$ \\
\hline$I B S-Q o L$ & & $\times$ & $\times$ & $\times$ & $\times$ \\
\hline
\end{tabular}

Fig. 2 Participant timeline

1) Are lost to follow-up

2) Become pregnant

3) Develop serious adverse event (SAE)

Participants can withdraw from this clinical trial at any time. The date and reason for withdrawal should be stated. If possible, all subjects withdrawing from the study should continue to be followed up regularly on a measurement schedule with a final assessment. Participants who discontinue early will not be replaced.

\section{Recruitment}

We will recruit participants through advertisements in newspapers and TV programs. Screening will be done by researchers. Informed consent will be obtained from eligible patients before randomization.

\section{Intervention}

Each patient will be scheduled for a total of 12 treatment sessions, $30 \mathrm{~min}$ for each session, two times a week over a 6-week period. The selection of acupuncture points is based on the traditional Chinese medicine (TCM) theory [24-26] and evidence-based clinical research [22]. According to the traditional Chinese medicine (TCM) theory, the spleen transforms the food digested by the stomach into essences. It helps to transport throughout the body, while the liver ensures the smooth flow of Qi, modifies the activity of internal organs (Zang Fu), and is highly related to the emoto-psychological stage. Therefore, most TCM experts agree that the weakness of the spleen and stomach is the basic pathogenesis of IBS, while the stagnant among the liver and spleen can also cause a serious symptom of IBS [27]. Therefore, the principles of treatment of IBS are regulating the Qi movement among the liver and spleen. Thus, the following acupuncture points are selected for the treatment (Tables 1 and 2).

\section{Acupuncture (AC) group}

Disposable acupuncture needles $(0.30 \mathrm{~mm}$ in diameter and $25-40 \mathrm{~mm}$ in length) are inserted at a depth of $10-30 \mathrm{~mm}$ obliquely into the scalp acupuncture points (Baihui, Toulinqi) or straightly into the body acupuncture points (Guanyuan, Zhongwan, Tianshu, Zusanli, Sanyinjiao, Taichong, Zhangmen). Electroacupuncture will be applied to the abdominal points at fast and dispersed waves through an electric needle stimulator (ES-160 6-Channel Programmable Electroacupuncture) for $30 \mathrm{~min}$. The intensity is adjusted to a level at which patients feel comfortable. The alternating stimulation is believed to produce maximal biochemical responses in the brain [28].

\section{Sham acupuncture (SAC) group}

Disposable acupuncture needles $(0.30 \mathrm{~mm}$ in diameter and $25-40 \mathrm{~mm}$ in length) are inserted at the same way as in the acupuncture group but on the sham acupuncture points (Sham-Baihui, Sham-Toulinqi, Sham-Guanyuan, Sham-Zhongwan, Sham-Tianshu, Sham-Zusanli, ShamSanyinjiao, Sham-Taichong, Sham-Zhangmen; Table 3, Figs. 3, 4, 5, 6, 7, 8, 9, and 10). The sham points are nonacupuncture points nor located on the meridians [29]. 
Table 1 Checklist for items in STRICTA 2010

Item

1. Acupuncture rationale

2. Details of needling

3. Treatment regimen

4. Other components of treatment

5. Practitioner background

6. Control or comparator interventions

Detail

1a) Style of acupuncture

Manual and electro-acupuncture base on traditional Chinese medicine theory and evidences from previous clinical trials.

1b) Reasoning for treatment provided, based on historical context, literature sources, and/or consensus methods, with references where appropriate

According to systematic reviews and clinical experiences of our principal investigator and co-investigators.

1c) Extent to which treatment was varied

Standard treatment is used. No variation of treatment among patients.

2a) Number of needle insertions per subject per session (mean and range where relevant) 15 needles.

2b) Names (or location if no standard name) of points used (uni/bilateral) Bilateral-Tianshu (ST25), Zusanli (ST36), Sanyinjiao (SP6), Toulinqi (GB15), Taichong (LR3), and Zhangmen (LR13). Unilateral—Baihui (GV20), Guanyuan (CV4), and Zhongwan (CV12).

2c) Depth of insertion, based on a specified unit of measurement, or on a particular tissue level 10-30 mm.

2d) Response sought (e.g., de qi or muscle twitch response)

De qi.

2e) Needle stimulation (e.g., manual, electrical)

Manual.

Electrical-dense-disperse waves with $50 \mathrm{~Hz}$ at 10 volts.

2f) Needle retention time

$30 \mathrm{~min}$.

2g) Needle type (diameter, length, and manufacturer or material)

Disposable stainless steel acupuncture needles (Dong Bang acupuncture needles, $0.25 \mathrm{~mm}$ in diameter and $40 \mathrm{~mm}$ in length with guide tube, manufactured by Dong Bang Acupuncture Inc. Korea).

3a) Number of treatment sessions

12 sessions.

3b) Frequency and duration of treatment sessions

2/week for 6 consecutive weeks.

4a) Details of other interventions administered to the acupuncture group (e.g., moxibustion, cupping, herbs, exercises, lifestyle advice)

None.

4b) Setting and context of treatment, including instructions to practitioners and information and explanations to patients

University clinics.

Participants will be informed about acupuncture treatment in the study as follows: "In this study, acupoints for IBS will be used based on related reports and clinical experience of our investigators."

5) Description of participating acupuncturists (qualification or professional affiliation, years in acupuncture practice, other relevant experiences)

Hong Kong-registered Chinese medicine practitioners having at least 3 years of clinical experience, who have undergone training and are able to provide identical acupuncture treatment in accordance with a pre-defined protocol.

6a) Rationale for the control or comparator in the context of the research question, with sources that justify this choice

To assess the efficacy and safety of electro-acupuncture compared to sham acupuncture.

6b) Precise description of the control or comparator. If sham acupuncture or any other type of acupuncture-like control is used, provide details as for items 1 to 3 above

- Style of acupuncture: sham acupuncture (needling at sham acupoints).

- Number of needle insertions per subject per session: 15 needles.

- Depth of insertion: 10-30 mm.

- Needle retention time: $30 \mathrm{~min}$.

- Needle type: the same as the treatment group.

- Number of treatment sessions: 12 sessions.

- Frequency and duration of treatment sessions: 2/week for 6 consecutive weeks.

Note: This checklist, which should be read in conjunction with the explanations of the STRICTA items, is designed to replace CONSORT 2010's item 5 when reporting an acupuncture trial 
Table 2 Acupuncture points

\begin{tabular}{|c|c|c|}
\hline $\begin{array}{l}\text { Acupuncture } \\
\text { point }\end{array}$ & Anatomical location & Function according to TCM \\
\hline Baihui (GV20) & 7 cun directly above the midpoint of the posterior hairline & Easing mental stress \\
\hline $\begin{array}{l}\text { Toulinqi } \\
\text { (GB15) }\end{array}$ & $\begin{array}{l}\text { Directly above the pupils when the eyes are looking straight ahead, } 0.5 \text { cun within the anterior } \\
\text { hairline, at the midpoint of the line connecting Shenting (GV24) and Touwei (ST8) }\end{array}$ & Tranquilizing the mind \\
\hline $\begin{array}{l}\text { Guanyuan } \\
\text { (CV4) }\end{array}$ & On the anterior midline, 3 cun below the umbilicus & Regulating qi \\
\hline $\begin{array}{l}\text { Zhongwan } \\
\text { (CV12) }\end{array}$ & On the anterior midline, 4 cun above the umbilicus & $\begin{array}{l}\text { Regulating the stomach to } \\
\text { smooth qi }\end{array}$ \\
\hline Tianshu (ST25) & 2 cun lateral to the umbilicus & $\begin{array}{l}\text { Regulating qi flow for } \\
\text { activating stagnancy }\end{array}$ \\
\hline Zusanli (ST36) & 3 cun below Dubi (ST35), one finger width lateral to the anterior crest of the tibia & $\begin{array}{l}\text { Strengthening the spleen and } \\
\text { stomach }\end{array}$ \\
\hline $\begin{array}{l}\text { Sanyinjiao } \\
\text { (SP6) }\end{array}$ & 3 cun above the medial malleolus, on the posterior border of the medial aspect of the tibia & $\begin{array}{l}\text { Strengthening the spleen and } \\
\text { stomach }\end{array}$ \\
\hline $\begin{array}{l}\text { Taichong } \\
\text { (LR3) }\end{array}$ & In the depression distal to the junction of the 1st and 2nd metatarsal bones & $\begin{array}{l}\text { Calming down the liver and } \\
\text { stop the pain }\end{array}$ \\
\hline $\begin{array}{l}\text { Zhangmen } \\
\text { (LR13) }\end{array}$ & On the lower border of the free end of the 11 th rib & $\begin{array}{l}\text { Spreading the liver qi and } \\
\text { stop the pain }\end{array}$ \\
\hline
\end{tabular}

\section{Outcomes}

The primary outcome is the difference between baseline and 8 weeks and 14 weeks after randomization on IBS Symptom Severity Scale (IBS-SSS).

The secondary outcomes include (1) the difference between baseline and 8 weeks and 14 weeks after randomization on Hamilton Depression Rating Scale (HAMD-17), (2) the difference between baseline and 8 weeks and 14 weeks after randomization on Clinical Global Impression-Severity (CGI-S) and Self-Rating Depression Scale (SDS), and (3) the difference between baseline and 8 weeks and 14 weeks after randomization on IBS Quality of Life (IBS-QoL). IBS-SSS has been validated as a classical monitor of the disease since 1997 and has been widely adopted across IBS researches [30]. The specific assessment schedule is in Fig. 2. Safety profiles will be assessed by determining the important adverse events reported on in every treatment and follow-up interviews.

\section{Assignment and blinding}

Simple, complete, non-sequential randomization numbers will be generated by the Random Allocation Software (Version 1.0.0), Isfahan, Iran, and kept by the principal investigator (PI). After the patient's eligibility is confirmed, patients will be randomized to $\mathrm{AC}$ arm or SAC arm for 6 weeks. Only the acupuncturists are allowed to know the patients' treatment. All other research members will be blind to the assignment. In

Table 3 Sham acupuncture points

\begin{tabular}{|c|c|}
\hline Sham points & Location \\
\hline Sham-Baihui (GV20) & 0.5 cun lateral from GV20 \\
\hline Sham-Toulinqi (GB15) & $\begin{array}{l}\text { At the midpoint of the line connecting GB15 and ST8 ( } 0.5 \text { cun above the hairline, at the corner of the forehead, } \\
4.5 \text { cun lateral from the midline), } 0.5 \text { cun within the anterior hairline }\end{array}$ \\
\hline Sham-Guanyuan (CV4) & In the mid-way of CV4 and ST28, 1 cun lateral to the anterior midline, 3 cun below the umbilicus \\
\hline Sham-Zhongwan (CV12) & In the mid-way of CV12 and ST21, 1 cun lateral to the anterior midline, 4 cun above the umbilicus \\
\hline Sham-Tianshu (ST25) & In the mid-way of ST25 and SP15, 3 cun lateral to the umbilicus \\
\hline Sham-Zusanli (ST36) & Horizontal to ST36, on the edge of the fibula \\
\hline Sham-Sanyinjiao (SP6) & $\begin{array}{l}\text { Horizontal to SP6, } 3 \text { cun above the depression between the tip of the medial malleolus and the tendo } \\
\text { calcaneus (KI3) }\end{array}$ \\
\hline Sham-Taichong (LR3) & $\begin{array}{l}\text { In the depression distal to the junction of the 3rd and 4th metatarsal bones, between the stomach and } \\
\text { gall bladder channel }\end{array}$ \\
\hline Sham-Zhangmen (LR13) & $\begin{array}{l}\text { In the mid-way of LR13 and ST23 horizontally, } 2 \text { cun above the umbilicus, on the outer edge of the rectus } \\
\text { abdomius }\end{array}$ \\
\hline
\end{tabular}




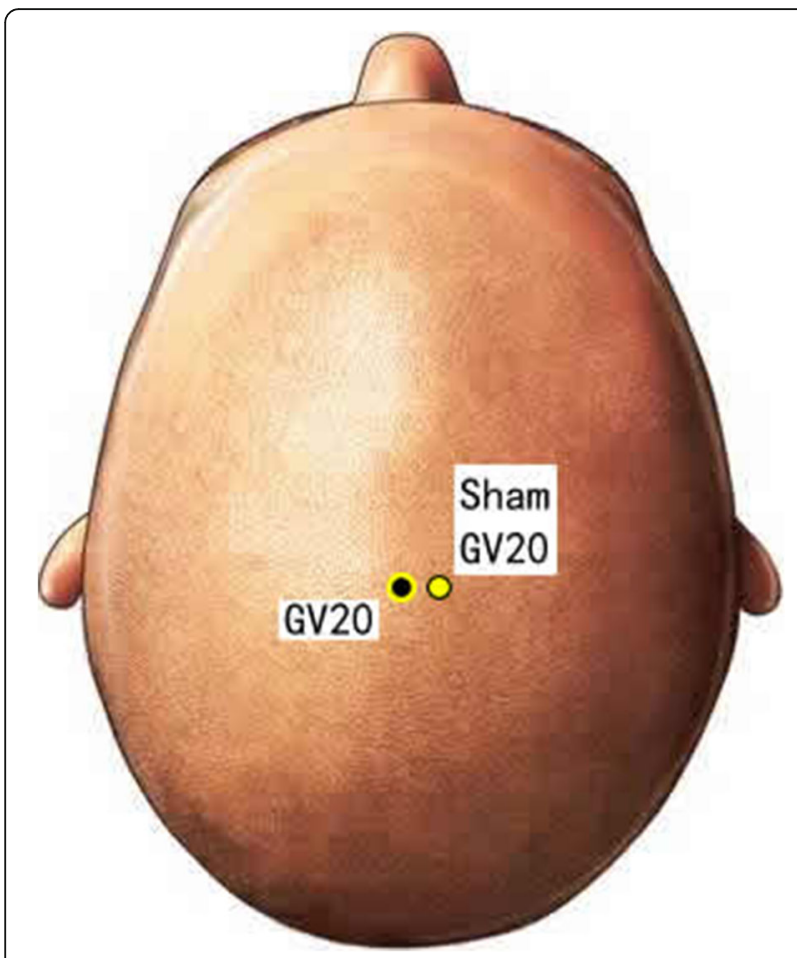

Fig. 3 Sham points: GV 20 and Sham GV20

addition, patients will also be blinded. They cannot visually detect sham or active acupuncture procedures.

\section{Sample size calculation}

According to a previous study, placebo is associated with high rates of resolution in a functional bowel disorder. Assuming that a difference of at least 30\% between acupuncture and placebo is needed for a clinically

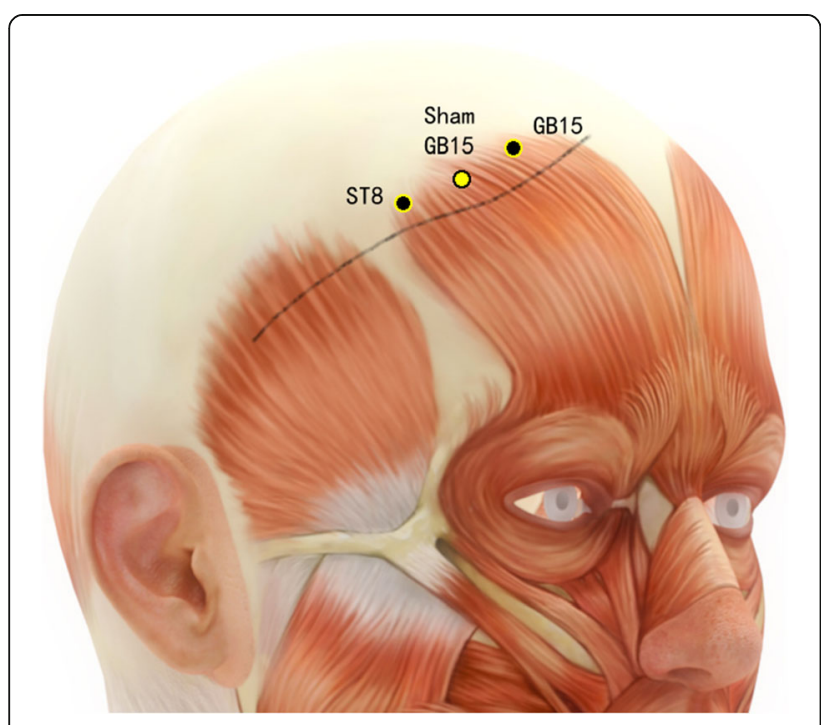

Fig. 4 Sham points: ST8, Sham GB15, and G15

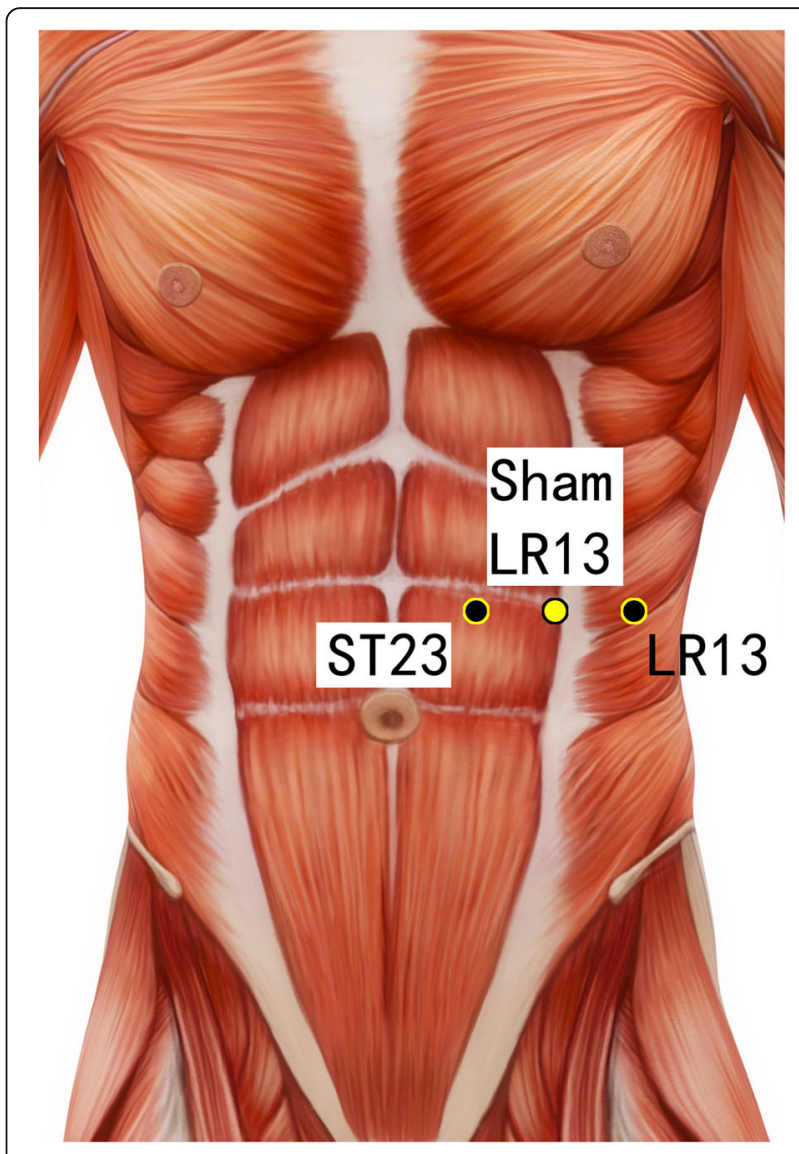

Fig. 5 Sham points: ST23, Sham LR13, and LR13

important outcome, 51 patients per treatment group were deemed sufficient to achieve $80 \%$ power in detecting a treatment difference, based on a two-sided $\chi^{2}$ test without continuity correction at a significance level of 0.025 (used to maintain the overall significance level at $5 \%)$. Further assuming a $15 \%$ dropout rate, we concluded that we needed to recruit a total of 120 patients (60 per arm) for this trial to ensure statistically significant results. The calculation was performed using the StudySize 3.0 software (V. Frolunda, Sweden).

\section{Statistical analysis}

All efficacy and safety analyses will be conducted according to the intention-to-treat (ITT) principle. Missing values will be imputed by the last-observation-carried forward method. The statistical analysis will be performed using the Statistical Packages of Social Sciences (SPSS) for Windows version 25.0. The statistical significance is defined as a two-sided $P$-value of $<0.05$. Baseline differences between the groups will be assessed with the use of Student's $t$-test for normally distributed continuous variables and the non-parametric MannWhitney $U$ test for non-normally distributed. For categorical variables, the chi-squared test or Fisher's exact 


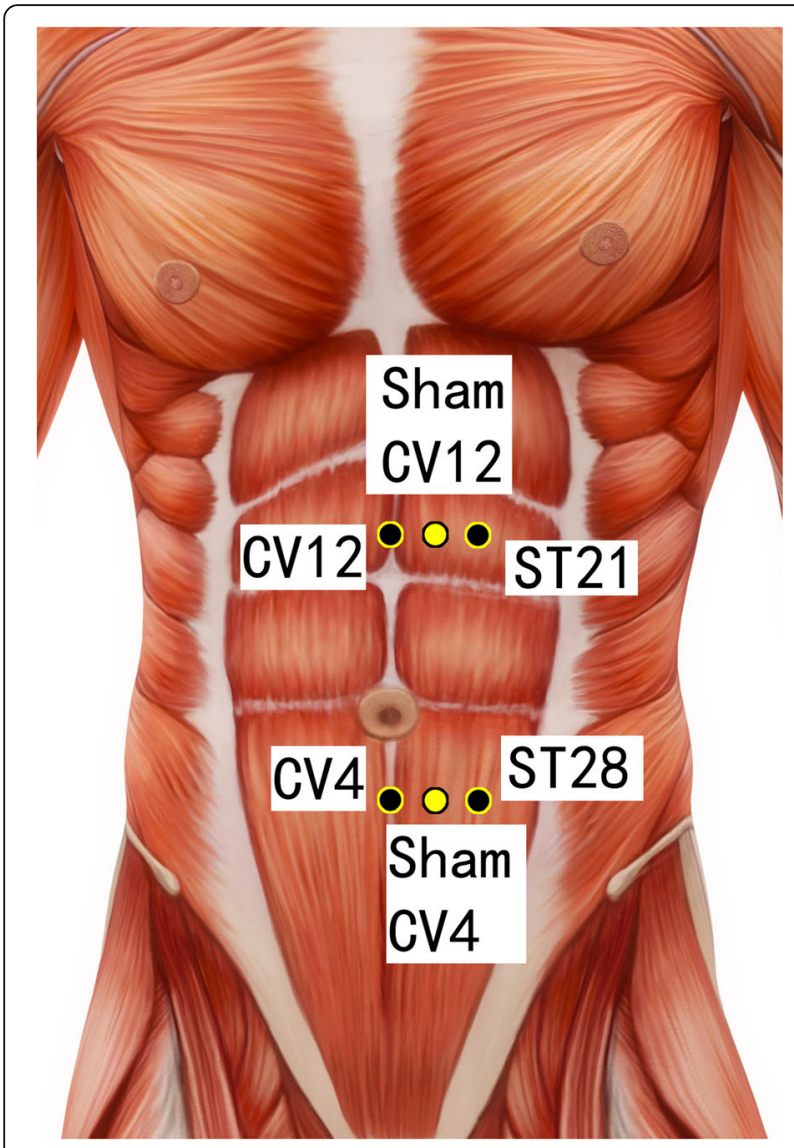

Fig. 6 Sham points: CV12, Sham CV12, ST21, CV4, Sham CV4, and ST28 test will be used. Changes in IBS-SSS, HAMD-17, SDS, and IBS-QoL at each evaluation time point from baseline will be analyzed using the ANCOVA model, followed by the Bonferroni $t$-test to detect the differences between the two groups at each time point. Incidence of adverse events will be examined using the chi-square test.

\section{Discussion and conclusion}

The study is a single-blinded, randomized controlled clinical trial to evaluate the efficacy and safety of acupuncture for IBS, including all subtypes: IBS-C, IBS-D, IBS-M, and IBS-U. In addition to the change of bowel habits, people with IBS frequently suffered from anxiety, and depression or mood change further worsens the IBS. Currently, there is not any study on acupuncture in treating IBS digestive-psychological symptoms; therefore, the scalp points and abdominal points are included in this clinical trial. It would be the first of such research.

If the outcome from this study shows improvement in IBS, we may expect a larger scale of study. Research on the integration of non-medication treatments can be

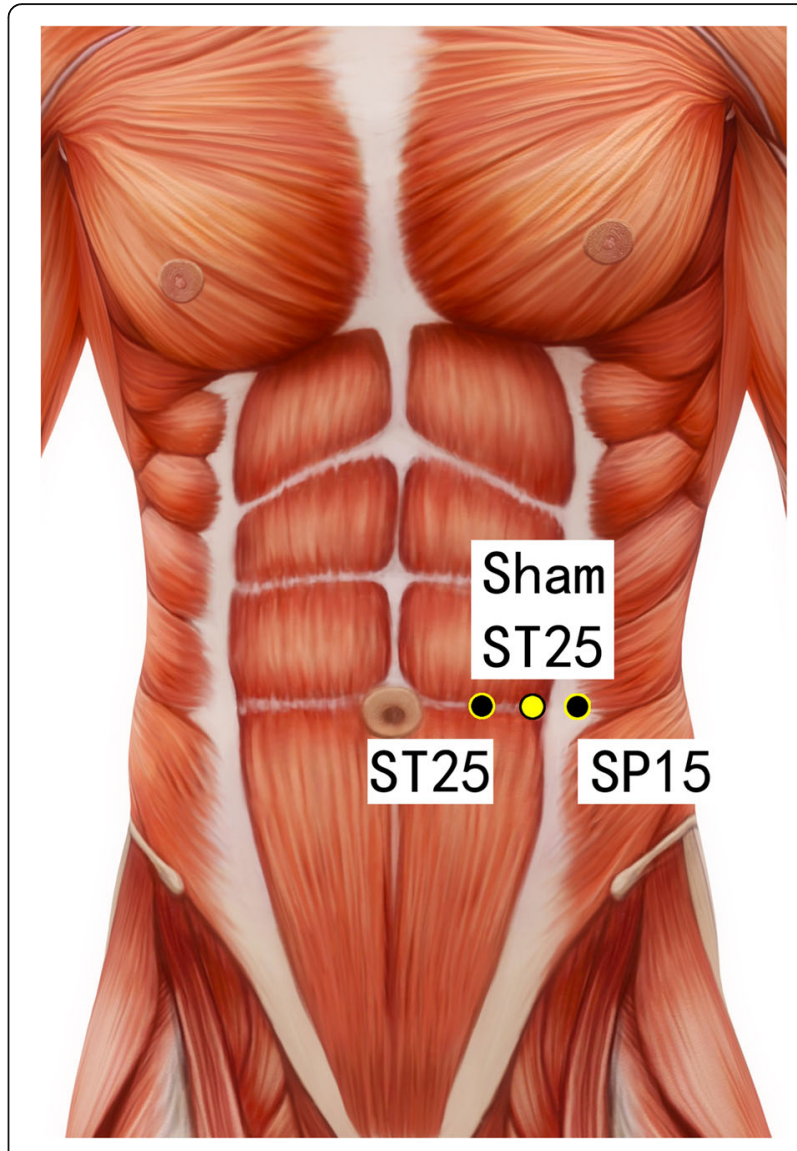

Fig. 7 Sham points: ST25, Sham ST25, and SP15

developed with the data from this clinical trial, such as electro-acupuncture with diet or lifestyle change, or integration of acupuncture and psychological cognitivebehavioral therapy.

The limitation of this study is that the selection of acupuncture points is standardized and utilized for every subject without syndrome differentiation. IBS can be differentiated into 4 subtypes, and each can be further subdivided with Chinese medicine syndrome differentiation such as spleen dysfunction, liver depression, kidney Yang deficiency, and intestinal heat dampness. Although dysfunction of the spleen system is the leading cause of the problem, point selection according to each subtype may optimize the effectiveness of acupuncture. In addition, the environmental factors and dietary factors of the participants exposed to may affect the study result. We would require participants to record their daily diet on a diet diary, but no dietary modification would be given in our study.

In conclusion, this study will provide the basis for the effectiveness and safety of electro-acupuncture on IBS global treatment and would explore the possibility of 

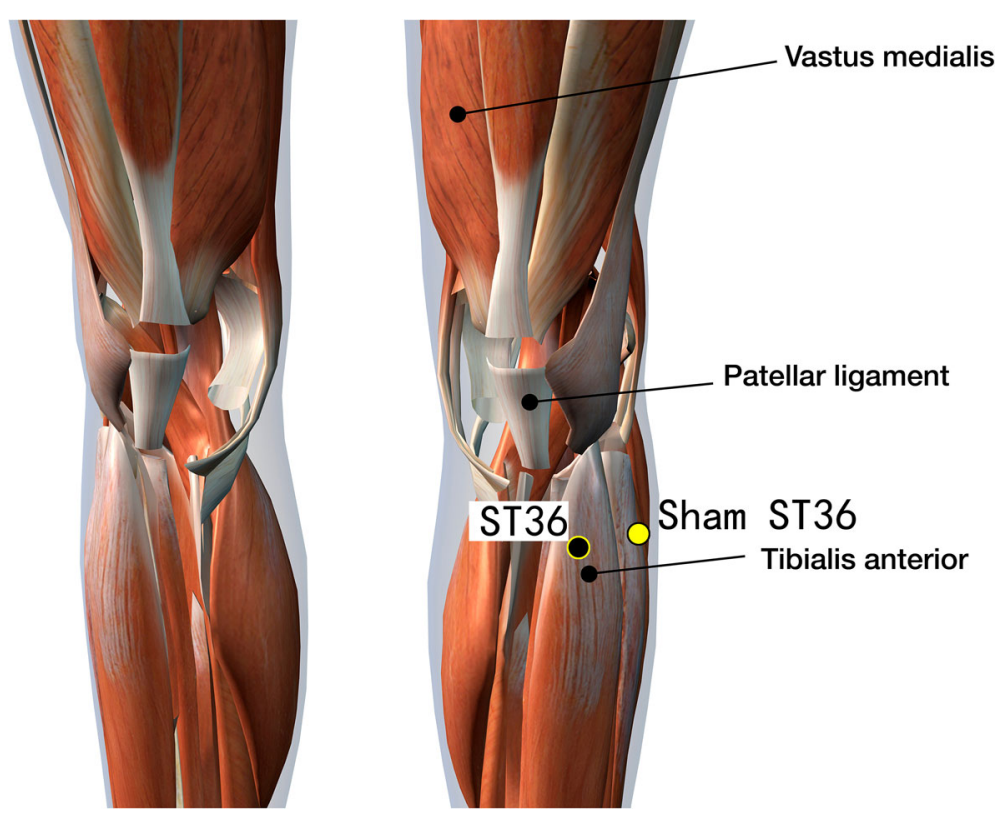

Fig. 8 Sham points: ST36 and Sham ST36

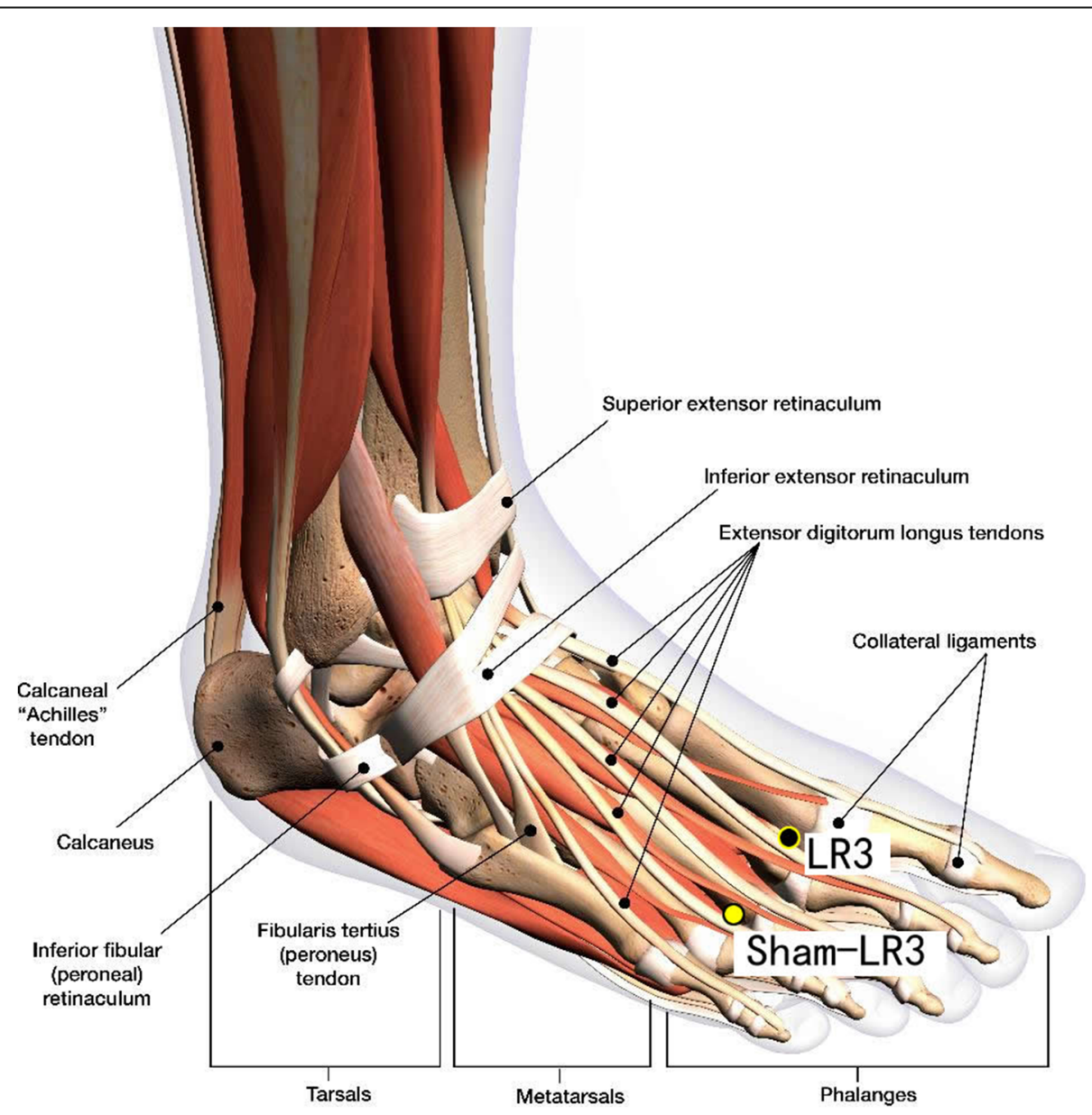

Fig. 9 Sham points: LR3 and Sham LR3 


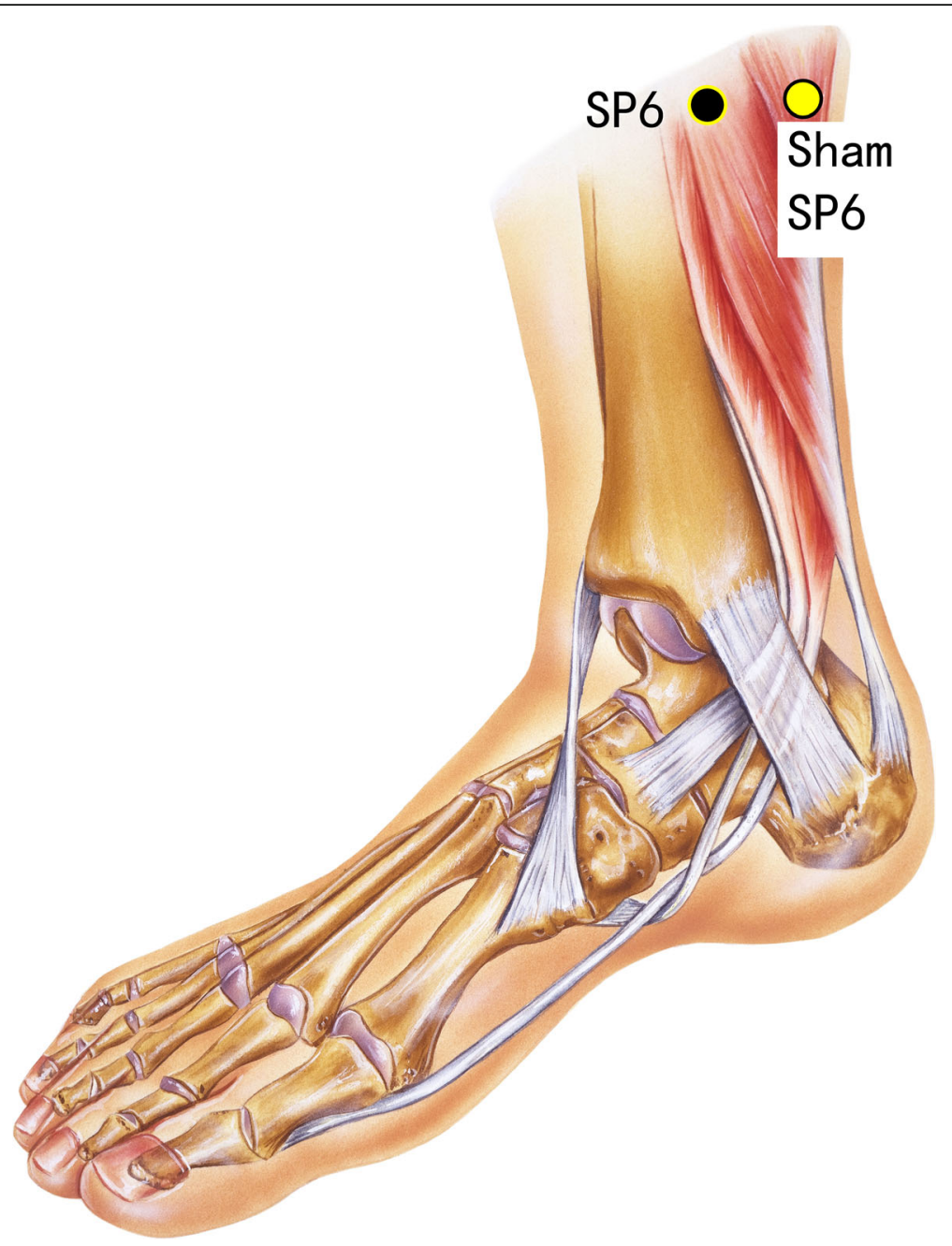

Fig. 10 Sham points: SP6 and Sham SP6

acupuncture in the integrative treatment of psychological conditions.

\section{Trial status}

A pilot study with a sample size of 20 (14.3\% of the total 140 participants, 10 in each arm) has been done to estimate the efficacy of this protocol and to observe the outcomes and any development of adverse events. The rate of recruitment for the pilot trial of 20 participants is $100 \%$. Another 120 participants will be recruited from August 2021. The study will be completed in July 2022. The schedule may be delayed with the COVID-19 pandemic which may be discouraging for patients joining any clinical trials. The problem would be mitigated with our fully vaccinated clinical staff and the growing vaccination rate in the Hong Kong population.

\section{Supplementary Information}

The online version contains supplementary material available at https://doi. org/10.1186/s13063-021-05563-4.

\author{
Additional file 1. IBS-SSS \\ Additional file 2. HAMD-17. \\ Additional file 3. CGI-S \\ Additional file 4. SDS. \\ Additional file 5. IBS-QOL. \\ Additional file 6. Consent form. \\ Additional file 7. Questionnaire with the Bristol Stool Form Scale.
}

\section{Acknowledgements}

We thank K.Y. and Betty Ho for sponsoring us. We also thank all the participants in this study.

\section{Authors' contributions}

LDZ and ZXB designed and supervised the study. LDZ and TFL drafted the manuscript. LDZ, TFL, and YZ enrolled the participants and conducted the 
treatments. LDZ, WY, and ZL analyzed the data. All authors read and approved the final manuscript.

\section{Funding}

This research was financially supported by the donation from K.Y. and Betty Ho. The sponsors have no role in designing and analyzing the study.

\section{Availability of data and materials}

The datasets used and analyzed during the current study are available from the corresponding author on reasonable request

\section{Declarations}

\section{Ethics approval and consent to participate}

This study protocol has been approved by the Hong Kong Baptist University Ethics Committee on the Use of Human Subjects for Teaching and Research (approval number: REC/18-19/0227).

Consent is obtained from every participant.

\section{Consent for publication}

Written informed consent was obtained from the patients for the publication of this manuscript and accompanying images.

\section{Competing interests}

The authors declare that they have no competing interests.

Received: 9 March 2021 Accepted: 24 August 2021

Published online: 15 September 2021

\section{References}

1. Drossman DA, Corazziari E, Delvaux M, et al., editors. Rome III: the functional gastrointestinal disorders. McLean: Degnon Associates; 2006.

2. Lovell RM, Ford AC. Global prevalence of and risk factors for irritable bowel syndrome: a meta-analysis. Clin Gastroenterol Hepatol. 2012;10(7):712-21 e4.

3. Lau EM, Chan FK, Ziea ET, et al. Epidemiology of irritable bowel syndrome in Chinese. Dig Dis Sci. 2002;47:2621-4.

4. Whitehead WE, Palsson O, Jones KR. Systematic review of the comorbidity of irritable bowel syndrome with other disorders: what are the causes and implications? Gastroenterology. 2002;122:1140-56.

5. American Gastroenterological Association. AGA technical review on irritable bowel syndrome. Gastroenterology. 2002;123:2108-31.

6. Drossman DA, Toner BB, Whitehead WE, et al. Cognitive-behavioral therapy versus education and desipramine versus placebo for moderate to severe functional bowel disorders. Gastroenterology. 2003;125:19-31.

7. Chang F-Y, Ching-Liang L. Treatment of irritable bowel syndrome using complementary and alternative medicine. J Chin Med Assoc. 2009:72:No 6

8. Liu Y-L, Liu J-S. Irritable bowel syndrome in China: a review on the epidemiology, diagnosis, and management. Chin Med J. 2021;134(12):1396401

9. Bhesania N, Cresci, Gail AM. A nutritional approach for managing irritable bowel syndrome. [Review]. Curr Opin Pediatr. 2017;29(5):584-91 10.

10. Flik CE, Bakker $L$, Laan $W$, et al. Systematic review: the placebo effect of psychological interventions in the treatment of irritable bowel syndrome. [Review]. World J Gastroenterol. 2017;23(12):2223-33.

11. NIH Consensus Conference. Acupuncture. JAMA. 1998:280:1518-24.

12. Kaptchuk TJ. Acupuncture: theory, efficacy, and practice. Ann Intern Med. 2002;136:374-83.

13. Stamuli $\mathrm{E}$, Bloor $\mathrm{K}, \mathrm{Mac}$ Pherson $\mathrm{H}$, et al. Cost-effectiveness of acupuncture for irritable bowel syndrome: findings from an economic evaluation conducted alongside a pragmatic randomised controlled trial in primary care. BMC Gastroenterol. 2012;12:149.

14. Ouyang $H$, Chen JDZ. Review article: therapeutic roles of acupuncture in functional gastrointestinal disorders. Aliment Pharmacol Ther. 2004;20: $831-41$.

15. Hakahashi T. Acupuncture for functional gastrointestinal disorders. J Gastroenterol. 2006:41:408-17.

16. Zheng $\mathrm{H}$, Li Y, et al. Electro-acupuncture for patients with diarrhea predominant irritable bowel syndrome or functional diarrhea: a randomized controlled trial. Medicine. 2016;95:24.
17. Zhang ZJ, Chen HY, Yip KC, et al. The effectiveness and safety of acupuncture therapy in depressive disorders: systematic review and metaanalysis. J Affect Disord. 2010;124:9-21.

18. Lee Y-T, Hu L-Y, et al. Risk of psychiatric disorders following irritable bowel syndrome: a nationwide population-based cohort study. PLoS One. 2015; 10(7):e0133283.

19. Lembo AJ, Conboy L, Kelley JM, Schnyer RS, McManus CA, Quilty MT, et al. A treatment trial of acupuncture in IBS patients. Am J Gastroenterol. 2009; 104:1489-97.

20. MacPherson H, Tilbrook H, Bland JM, Bloor K, Brabyn S, Cox H, et al. Acupuncture for irritable bowel syndrome: primary care based pragmatic randomised controlled trial. BMC Gastroenterol. 2012;12:150.

21. Manheimer E, Wieland LS, Cheng K, Li SM, Shen X, Berman BM, et al. Acupuncture for irritable bowel syndrome: systematic review and metaanalysis. Am J Gastroenterol. 2012;107(6):835-47.

22. Schneider A, Enck $P$, Streitberger $K$, et al. Acupuncture treatment in irritable bowel syndrome. Gut. 2006:55(5):649-54.

23. Manheimer E, Cheng K, Wieland LS, et al. Acupuncture for treatment of irritable bowel syndrome: Wiley; The Cochrane Collaboration. The Cochrane Library. 2012, Issue 5

24. Drossman D, Chang L, Chey W, Kellow J, Task J, Whitehead W. Rome IV Functional gastrointestinal disorder-disorder of gut-brain interaction. Gastroenterology 2016;150:1257-61

25. China Academy of Chinese Medical Sciences. Evidence-based guidelines of clinical practice in Chinese medicine internal medicine: China Press of Traditional Chinese Medicine; 2011. [Chinese]

26. Shen $X Y$, Wang $H$, editors. Acupuncture and moxibustion: People's Medical Publishing House; 2007.

27. Xiao HT, Zhong L, Tsang SW, Lin ZS, Bian ZX. Traditional Chinese medicine formulas for irritable bowel syndrome: from ancient wisdoms to scientific understandings. Am J Chin Med. 2015;43(1):1-23.

28. Han JS. Acupuncture: neuropeptide release produced by electrical stimulation of different frequencies. Trends in Neurosciences. 2003:26:17-22.

29. Liu Z, Yan S, Wu J, He L, Li N, Dong G, et al. Acupuncture for chronic severe functional constipation: a randomized, controlled trial. Annals of Internal Medicine. 2016;165(11): 761-70

30. Francis CY, Morris J, Whorwell PJ. The irritable bowel severity scoring system: a simple method of monitoring irritable bowel syndrome and its progress. Aliment Pharmacol Ther. 1997;11(2):395-402.

\section{Publisher's Note}

Springer Nature remains neutral with regard to jurisdictional claims in published maps and institutional affiliations.

Ready to submit your research? Choose BMC and benefit from:

- fast, convenient online submission

- thorough peer review by experienced researchers in your field

- rapid publication on acceptance

- support for research data, including large and complex data types

- gold Open Access which fosters wider collaboration and increased citations

- maximum visibility for your research: over $100 \mathrm{M}$ website views per year

At BMC, research is always in progress.

Learn more biomedcentral.com/submission 